九州大学学術情報リポジトリ

Kyushu University Institutional Repository

\title{
Low-grade dedifferentiated liposarcoma of the neck: Magnetic resonance imaging and pathological correlation
}

\section{Endo, Makoto}

Department of Anatomic Pathology, Graduate School of Medical Sciences, Kyushu University

Oda, Yoshinao

Department of Anatomic Pathology, Graduate School of Medical Sciences, Kyushu University

Harimaya, Katsumi

Department of Orthopaedic Surgery, Graduate School of Medical Sciences, Kyushu University

Tamiya, Sadafumi

Department of Anatomic Pathology, Graduate School of Medical Sciences, Kyushu University 他

http://hdl. handle. net/2324/26581

出版情報：Journal of Orthopaedic Science. 15 (1)，pp.148-152，2010-01-01. Springer Japan バージョン：

権利関係: (C) The Japanese Orthopaedic Association 
Low-grade dedifferentiated liposarcoma of the neck: magnetic resonance imaging and pathological correlation

\section{Running title: Low-grade DDLS}

Makoto Endo $^{1}$, Yoshinao Oda ${ }^{1}$, Katsumi Harimaya ${ }^{2}$, Sadafumi Tamiya $^{1}$, Hidetaka Yamamoto ${ }^{1}$, Kenichi Kohashi ${ }^{1}$, Shuichi Kurihara ${ }^{1}$, Nokitaka Setsu ${ }^{1}$, Suguru Matsuura ${ }^{1}$, Hiroshi Matono ${ }^{1}$, Shuichi Matsuda ${ }^{2}$, Yukihide Iwamoto ${ }^{2}$, Masazumi Tsuneyoshi ${ }^{1}$

${ }^{1}$ Department of Anatomic Pathology, Graduate School of Medical Sciences, Kyushu University, Fukuoka, Japan

${ }^{2}$ Department of Orthopaedic Surgery, Graduate School of Medical Sciences, Kyushu University, Fukuoka, Japan

Correspondence to: Yoshinao Oda, MD, $\mathrm{PhD}$

Department of Anatomic Pathology

Graduate School of Medical Sciences

Kyushu University, Fukuoka, Japan

3-1-1 Maidashi, Higashi-ku, Fukuoka 812-8582

Japan

E-mail: oda@surgpath.med.kyushu-u.ac.jp

Tel: +81-92-642-6061

Fax: +81-92-642-5968 


\section{Introduction}

Dedifferentiated liposarcoma is defined as a malignant adipocytic neoplasm showing a transition from well-differentiated liposarcoma to nonlipogenic sarcoma. ${ }^{1}$ In most cases, the nonlipogenic component presents a high-grade morphology, however, the concept of lowgrade dedifferentiation has increasingly been recognized in recent years. ${ }^{2,3}$ Radiologically, especially with magnetic resonance imaging (MRI), a typical presentation of dedifferentiated liposarcoma is the coexistence of fatty and nonfatty components, so that a radiological diagnosis of ordinary (high-grade) dedifferentiated liposarcoma is not difficult in most cases. ${ }^{4}$ On the other hand, the radiological findings of low-grade dedifferentiated cases have not been investigated at all. Dedifferentiated component of (high-grade) dedifferentiated liposarcoma usually presents low intensity on T1-weighted and heterogeneous high intensity on T2-weighted images. However, we experienced a case where the dedifferentiated component showed low to intermediate intensity on both T1- and T2-weighted MR images. In this report, we present a case of low-grade dedifferentiated liposarcoma of the neck with emphasis on the correlation between MRI and pathological findings. We also discuss the importance of recognizing the radiological findings of such a case. 


\section{Case report}

A 48-year-old Japanese male was referred to our hospital with the complaint of a soft tissue mass and dull pain in the neck region. He had first recognized the mass 2 years earlier, and since then it had gradually grown. A clinical examination revealed an ill-defined, elastic soft and nontender mass in the posterolateral aspect of the neck. All laboratory data were within the normal ranges.

Concerning the imaging studies, MRI showed a multinodular soft tissue mass located within the right semispinalis muscle (Fig. 1). The main portion of the mass presented high intensity on both T1- (TR/TE, 517/10) and T2-weighted (TR/TE, 3700/105) images, and the high intensity was suppressed on the fat-suppressed T1-weighted images (TR/TE, 600/9) (Fig. 1B-D arrowheads). The central area of the mass was different in appearance, presenting low to intermediate intensity on both T1- and T2-weighted images, similar to the intensity surrounding muscles showed (Fig. 1B, C arrows). Following the administration of an intravenous gadolinium-containing contrast agent, a part of the central intervening area was enhanced (Fig. 1D). Based on these radiological features, intramuscular lipoma or well differentiated liposarcoma was considered as a preoperative diagnosis, even though enhancement effect is unusual for them. Additionally, especially concerned about enhancement effect on the post contrast MRI, a possibility of dedifferentiated liposarcoma could not be denied, even though T2-weighted image is unusual for dedifferentiated liposarcoma. We considered that marginal resection would be impossible because of the infiltrating growth pattern of the tumor on MRI, even if the tumor was benign. Therefore, we decided to perform a wide resection of the tumor without a biopsy.

The tumor was resected with the right semispinalis muscle and its fascia. Grossly, the maximum cut surface of the tumor revealed two different components (Fig. 2). The tumor was composed of a central whitish area (arrows) and a peripheral yellowish area 
(arrowheads), the latter of which was infiltrating the surrounding skeletal muscle. The central whitish component corresponded to the central intervening area on the MRI, and the peripheral yellowish component corresponded to the fatty area on the MRI. The tumor measured $5 \mathrm{~cm}$ at greatest diameter.

Histologically, the whitish area was mainly composed of spindle cells and fibrous stroma, and the yellowish area was composed of adipocytic cells (Fig. 3A). The transition between these components was abrupt. In the central area, spindle-shaped tumor cells proliferated in a fascicular pattern associated with abundant dense fibrous stroma (Fig. 3B). Atypical spindle or polygonal cells with enlarged nuclei were scattered in some parts of the spindle cell component. Massive tumor necrosis was also observed. Mitotic figures were inconspicuous. Focally, massive lymphoplasmacytic infiltration with immature vessels was recognized in the spindle cell component. In contrast, the peripheral area was composed of adipocytic proliferation with significant variation in cell size (Fig. 3C). Lipoblasts, some of which were multivacuolated, were scattered in the adipocytic area. An invasive growth pattern into the surrounding skeletal muscles was detected at the periphery. Immunohistochemically, the tumor cells were positive for CDK4 in both the adipocytic and spindle cell areas (Fig. 3D). Additionally, in the spindle cell area, the tumor cells also showed focal immunoreactivity for MDM2, alpha-smooth muscle actin, and desmin. The Ki-67 (MIB-1) labeling index was 5\% in the spindle cell area. A diagnosis of low-grade dedifferentiated liposarcoma was established. A distribution of each adipocytic and dedifferentiated component corresponded to the gross appearance of yellowish- and whitish-colored area.

As of 1 year after surgery, the patient has remained free of any evidence of local recurrence or metastasis.

The patient and his family were informed that data from the case would be submitted for publication, and they agreed. 


\section{Discussion}

Dedifferentiated liposarcoma was originally described as the coexistence of well-differentiated liposarcoma and high-grade sarcoma. ${ }^{5}$ However, the concept of low-grade dedifferentiation has increasingly been recognized in the past decade. ${ }^{2,3}$ Several previous reports described clinicopathological findings of low-grade dedifferentiated liposarcoma, ${ }^{2,3,6}$ but the radiological features have not been investigated at all. In this report, we present a case of low-grade dedifferentiated liposarcoma that shows peculiar radiological features compared to those of ordinary (high-grade) dedifferentiated liposarcoma.

Radiologically, a typical presentation of dedifferentiated liposarcoma is the coexistence of fatty and non-fatty solid components. ${ }^{4}$ On MRIs, the fatty component shows high intensity on both T1- and T2-weighted images, whereas the nonfatty component shows low intensity on T1-weighted and heterogeneous high intensity on T2-weighted images. Based on this finding, the radiological diagnosis of dedifferentiated liposarcoma is not difficult in most cases. In the present case, the nonfatty component shows low to intermediate intensity on both T1- and T2-weighted images, similar to the intensity surrounding muscles showed. A multinodular adipocytic appearance with a thick intervening structure and infiltrating margin mimicked intramuscular lipoma or well differentiated liposarcoma, ${ }^{7,8}$ although enhancement on the contrast enhanced MRI with gadolinium-containing contrast agent indicated dedifferentiation of the central portion. We have reaffirmed that the contrast enhanced MRI is essential in the evaluation of lipogenic tumor with an intervening area, even if intramuscular lipoma or well-differentiated liposarcoma is suspected.

The MR findings of the present case can be explained by its pathological findings. The main portion of dedifferentiated component in this case is characterized by abundant dense fibrous stroma, low to intermediate cellularity, and fewer blood vessels. In addition, fibrous tissue replaces massive tumor necrosis. Dense fibrous tissue shows low to intermediate 
intensity on T2-weighted images, whereas high-grade sarcoma usually shows heterogeneous high intensity due to variable histological patterns. On the other hand, immature vessels which were scattered in dedifferentiated component are reflected in mild enhancement on post contrast MRI. In summary, the peculiar radiological finding on MRI is based on low-grade dedifferentiation and scattered immature vessels on the pathological examination.

Dedifferentiated liposarcoma occurs most commonly in the retroperitoneum, followed by deep soft tissue of the extremities, spermatic cord, trunk, and head and neck. ${ }^{1}$ According to a large-scale analysis of 155 cases, ${ }^{2}$ only one case is located in the head and neck region. Our experience also shows that dedifferentiated liposarcoma of the neck region is rarely seen. ${ }^{9,10}$ Because the present case occurred in a rare location, the preoperative diagnosis of dedifferentiated liposarcoma was all the more difficult.

As for the treatment, the tumor was resected with the semispinalis muscle and its fascia. Pathological examination revealed that the tumor was resected with a wide margin. We don't plan to do any postoperative adjuvant therapies because the tumor was resected completely and the dedifferentiated component showed low-grade morphology. The patient is being followed closely at our hospital, and has remained free of any evidence of local recurrence or metastasis as of 1 year after surgery. The patient is scheduled to receive long-term follow-up at our hospital.

The pathological differential diagnoses in this case include the sclerosing or spindle cell type of well-differentiated liposarcoma. Spindle cell proliferation arranged in fascicular fashion and immunohistochemical positivity for CDK4 and MDM2 are common findings of both of these types of well-differentiated liposarcoma and dedifferentiated liposarcoma., 11-13 However, the present case shows a clear transition from well-differentiated liposarcoma to nonlipogenic sarcoma, and spindle cells don’t intermingle with adipocytes. Based on these pathological features, this case can evidently be diagnosed as dedifferentiated liposarcoma. Low-grade dedifferentiation in dedifferentiated liposarcoma was advocated by Henricks 
et al. ${ }^{2}$ and Elgar et al. ${ }^{3}$ in 1997. According to their series, low-grade dedifferentiation is characterized most often by the presence of uniform fibroblastic spindle cells with mild nuclear atypia, often organized in a fascicular pattern and exhibiting intermediate cellularity. Our case completely meets these criteria for low-grade dedifferentiated liposarcoma.

In summary, we present a case of low-grade dedifferentiated liposarcoma of the neck with emphasis on the correlation between radiological and pathological findings. Although such case is rare, misdiagnosis can lead to an improper choice of treatment, such as long-time observation or marginal resection. Recognition of these radiological and pathological findings of low-grade dedifferentiated liposarcoma may aid in the assessment of patients with lipogenic tumor. 


\section{References}

1. Dei Tos AP, Pedeutour F. Well differentiated liposarcoma and Dedifferentiated liposarcoma. In: Fletcher CDM, Unni KK, Mertens F, editors. World Health Organization Classification of Tumours Pathology and Genetics of Tumours of Soft Tissue and Bone. Lyon: IARC Press, 2002: 35-9.

2. Henricks WH, Chu YC, Goldblum JR, Weiss SW. Dedifferentiated liposarcoma: a clinicopathological analysis of 155 cases with a proposal for an expanded definition of dedifferentiation. Am J Surg Pathol 1997; 21:271-81.

3. Elgar F, Goldblum JR. Well-differentiated liposarcoma of the retroperitoneum: a clinicopathologic analysis of 20 cases, with particular attention to the extent of low-grade dedifferentiation. Mod Pathol 1997; 10:113-20.

4. Kransdorf MJ, Meis JM, Jelinek JS. Dedifferentiated liposarcoma of the extremities: imaging findings in four patients. AJR Am J Roentgenol 1993; 161:127-30. 5. Evans HL. Liposarcoma: a study of 55 cases with a reassessment of its classification. Am J Surg Pathol 1979; 3:507-23.

6. Fukunaga M. Histologically low-grade dedifferentiated liposarcoma of the retroperitoneum. Pathol Int 2001; 51:392-5.

7. Nishida J, Morita T, Ogose A, Okada K, Kakizaki H, Tajino T, Hatori M, Orui H, Ehara S, Satoh T, Shimamura T. Imaging characteristics of deep-seated lipomatous tumors: intramuscular lipoma, intermuscular lipoma, and lipoma-like liposarcoma. J Orthop Sci 2007; 12:533-41.

8. Matsumoto K, Hukuda S, Ishizawa M, Chano T, Okabe H. MRI findings in intramuscular lipomas. Skeletal Radiol 1999; 28:145-52.

9. Takahira T, Oda Y, Tamiya S, Yamamoto H, Kobayashi C, Izumi T, Ito K, Iwamoto Y, Tsuneyoshi M. Alterations of the RB1 gene in dedifferentiated liposarcoma. Mod Pathol 
$2005 ; 18: 1461-70$.

10. Sakamoto A, Oda Y, Adachi T, Saito T, Tamiya S, Iwamoto Y, Tsuneyoshi M.

Beta-catenin accumulation and gene mutation in exon 3 in dedifferentiated liposarcoma and malignant fibrous histiocytoma. Arch Pathol Lab Med 2002; 126:1071-8.

11. Binh MB, Garau XS, Guillou L, Aurias A, Coindre JM. Reproducibility of MDM2 and CDK4 staining in soft tissue tumors. Am J Clin Pathol 2006; 125:693-7.

12. Binh MB, Sastre-Garau X, Guillou L, de Pinieux G, Terrier P, Lagace R, Aurias A, Hostein I, Coindre JM. MDM2 and CDK4 immunostainings are useful adjuncts in diagnosing well-differentiated and dedifferentiated liposarcoma subtypes: a comparative analysis of 559 soft tissue neoplasms with genetic data. Am J Surg Pathol 2005; 29:1340-7.

13. Dei Tos AP, Mentzel T, Newman PL, Fletcher CD. Spindle cell liposarcoma, a hitherto unrecognized variant of liposarcoma. Analysis of six cases. Am J Surg Pathol 1994; $18: 913-21$ 


\section{Figure legends}

Fig. 1A-D. Coronal T2-weighted (A, TR/TE, 3000/98) MRI of the neck showing a multinodular high-intensity mass separated by intervening low-intensity band. Axial MRI shows the mass within the right semispinalis muscle mainly presenting high intensity on both T1-weighted (B, TR/TE, 517/10) and T2-weighted (C, TR/TE, 3700/105) images (arrowheads). The high-intensity area is separated by the intervening central area presenting low to intermediate intensity on both T1- and T2-weighted images, similar to the intensity surrounding muscles show (arrows). Following the administration of an intravenous gadolinium-containing contrast agent, enhancement is seen in a part of the intervening central area, and the high intensity of the main portion is suppressed on the fat-suppressed T1-weighted (D, TR/TE, 600/9) images.

Fig. 2A. Gross appearance of the tumor corresponding to the axial view of MRI. The upside of the figure corresponds to the superficial side of the body, and the underside of the figure corresponds to the deep side of the body. The cut surface reveals two different components: a central whitish area (arrows) and a peripheral yellowish area (arrowheads). The central whitish component corresponds to the bandlike area on MRI, and the peripheral yellowish component corresponds to the fatty area on MRI. B Schematic illustration of the tumor. The central horizontal-striped area represents the central whitish component, and the vertical-striped area represents the yellowish fatty component. The oblique-striped area represents semispinalis muscle.

Fig. 3A-D. Histological findings of the tumor. A The central whitish area is mainly composed of spindle cells, and the peripheral yellowish area is composed of adipocytic cells. The transition between components is abrupt. B In the central area, spindle-shaped tumor cells 
proliferate in a fascicular pattern associated with abundant fibrous stroma and lymphoplasmacytic infiltration. $\mathbf{C}$ The peripheral area is composed of adipocytic proliferation with significant variation in cell size. D Immunohistochemically, the tumor cells are positive for CDK4 in the spindle cell area. 
Fig. 1A

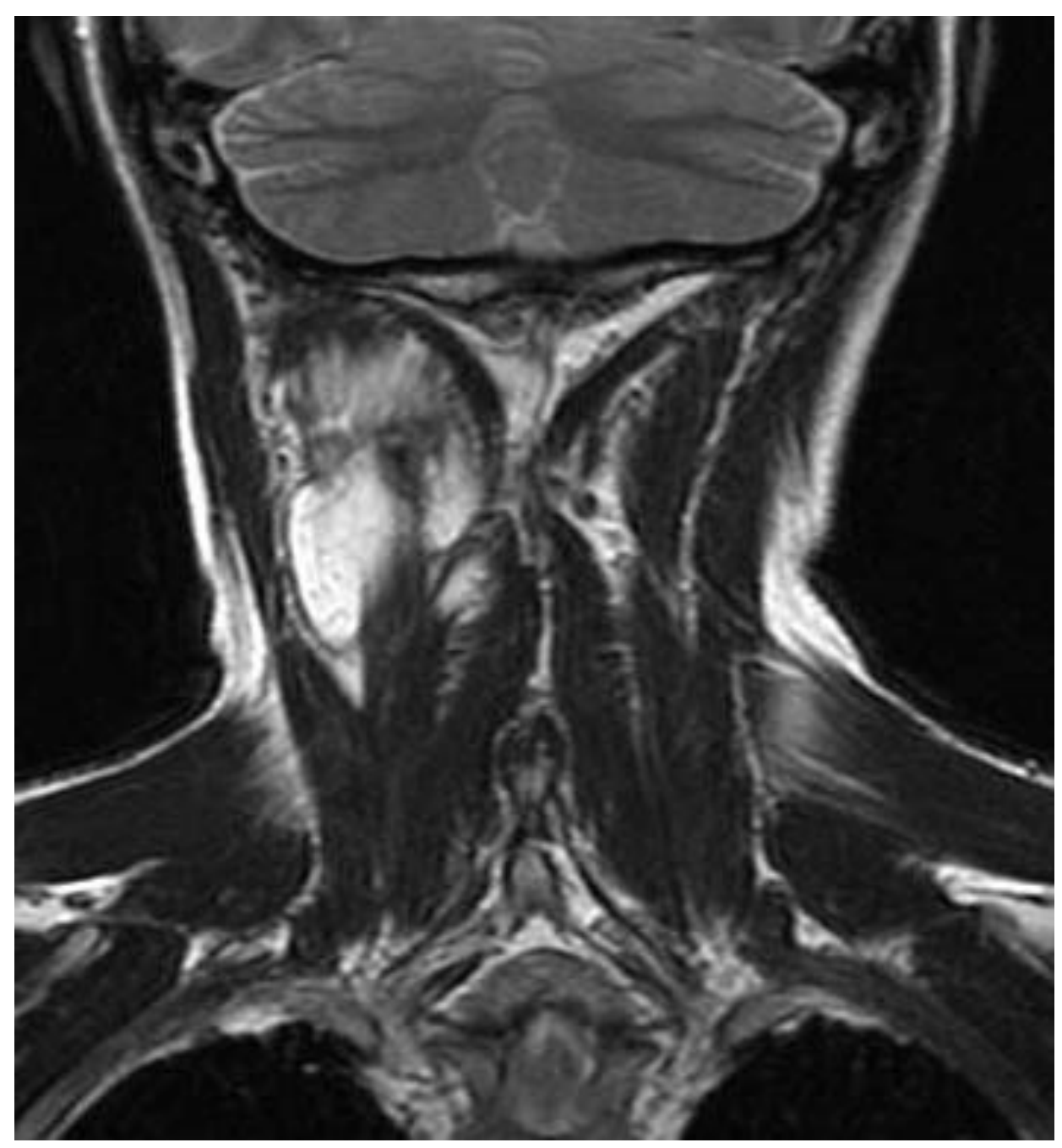


Fig. 1B

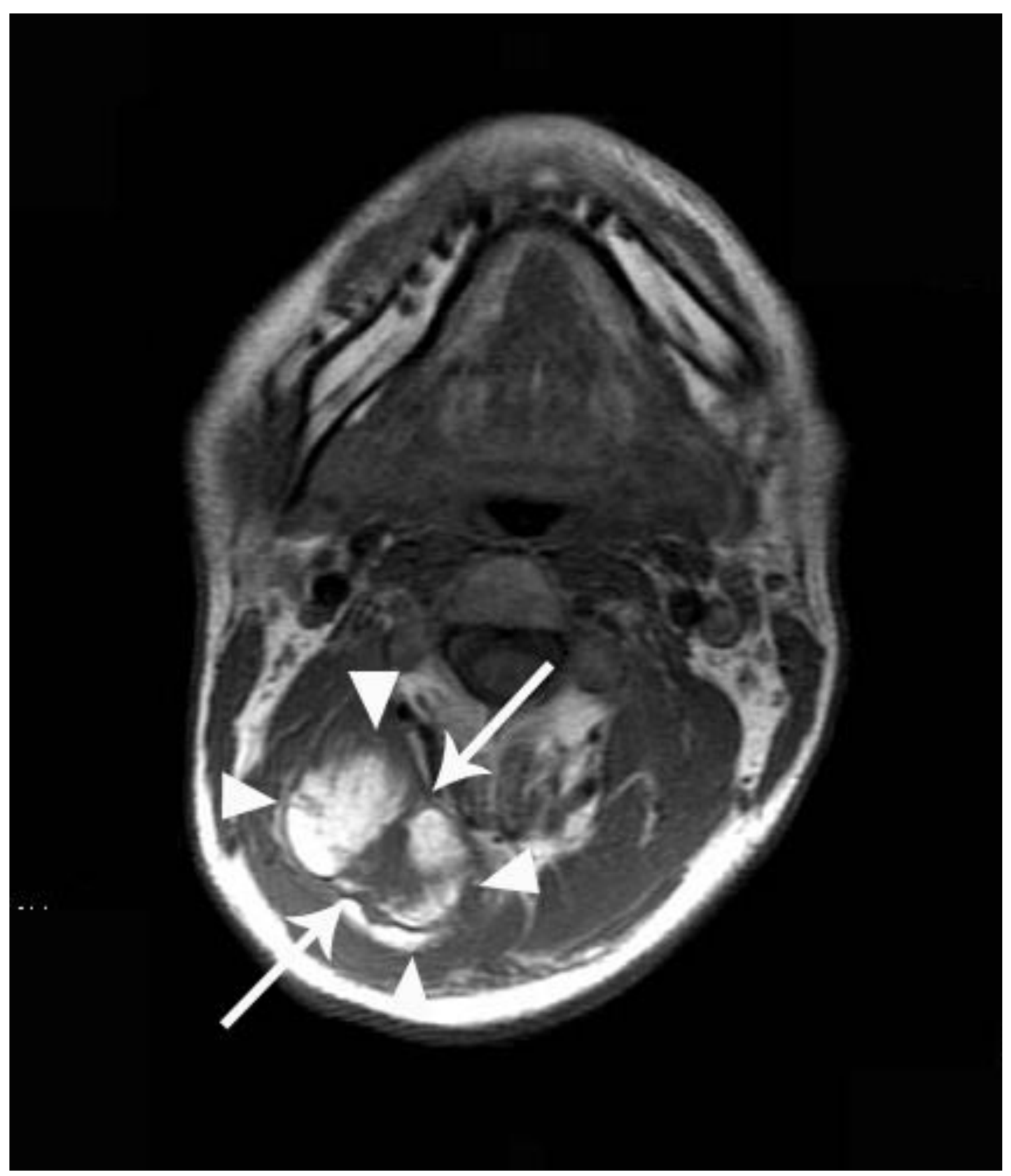


Fig. 1C

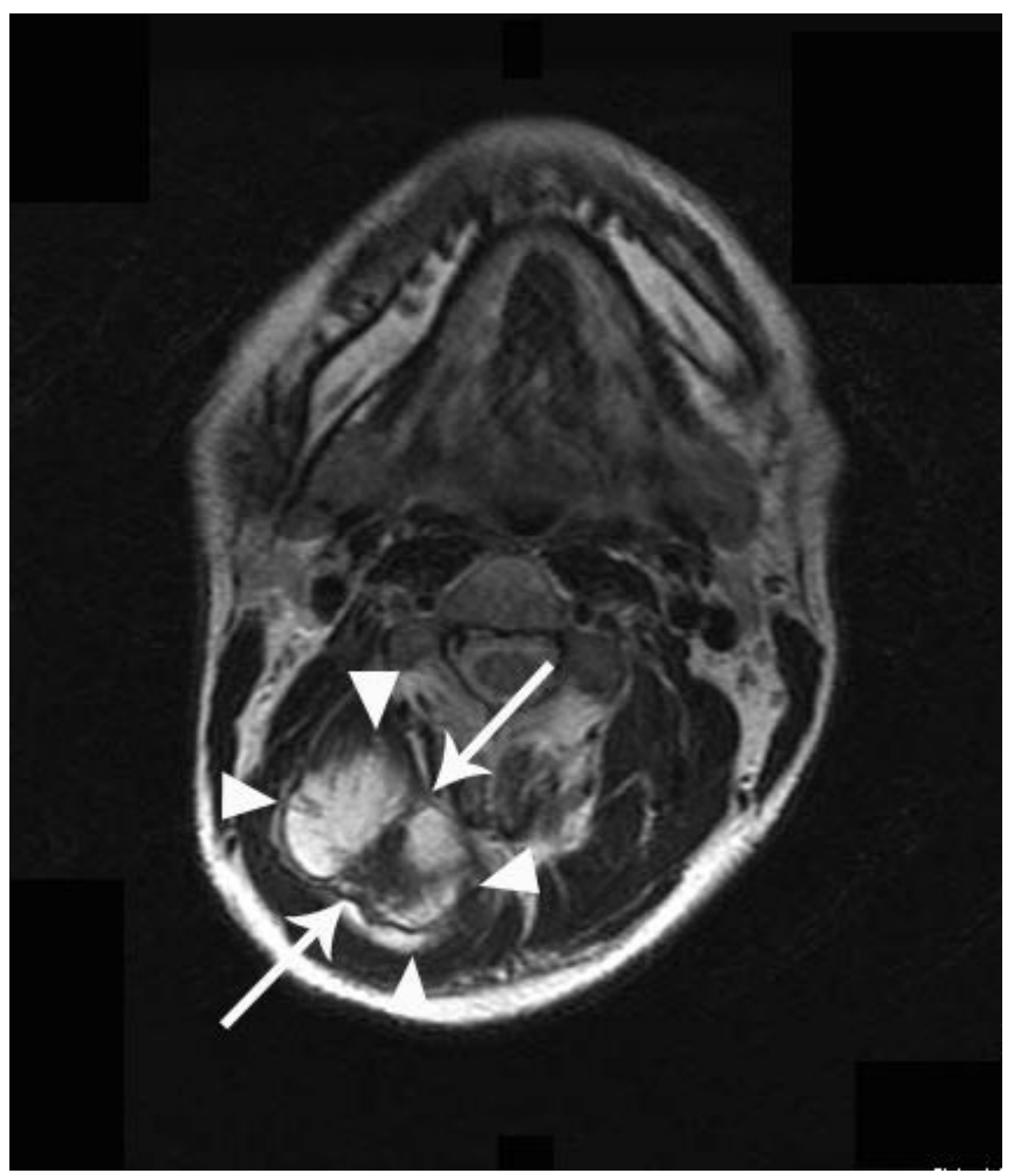


Fig. 1D

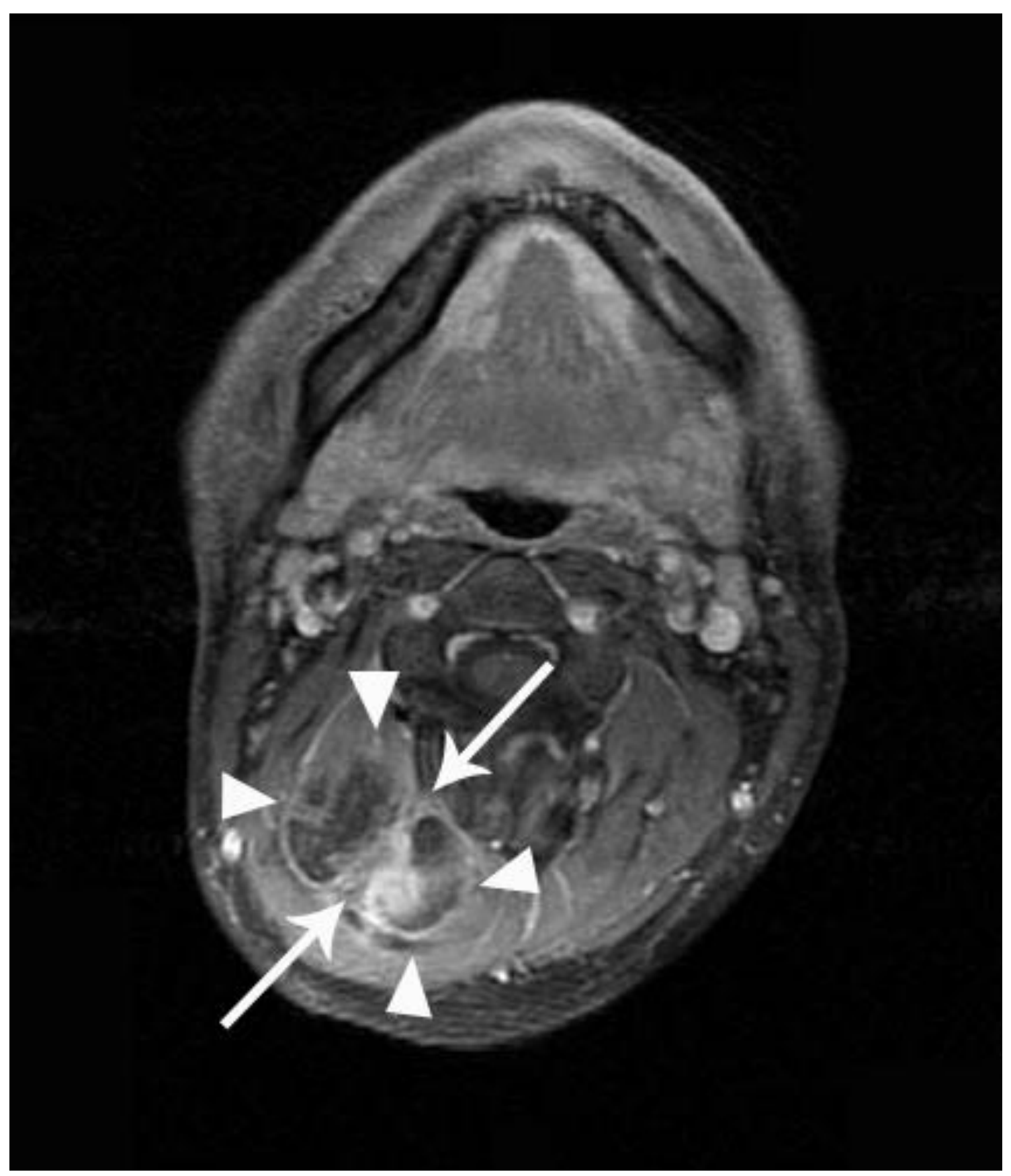


Fig. 2A

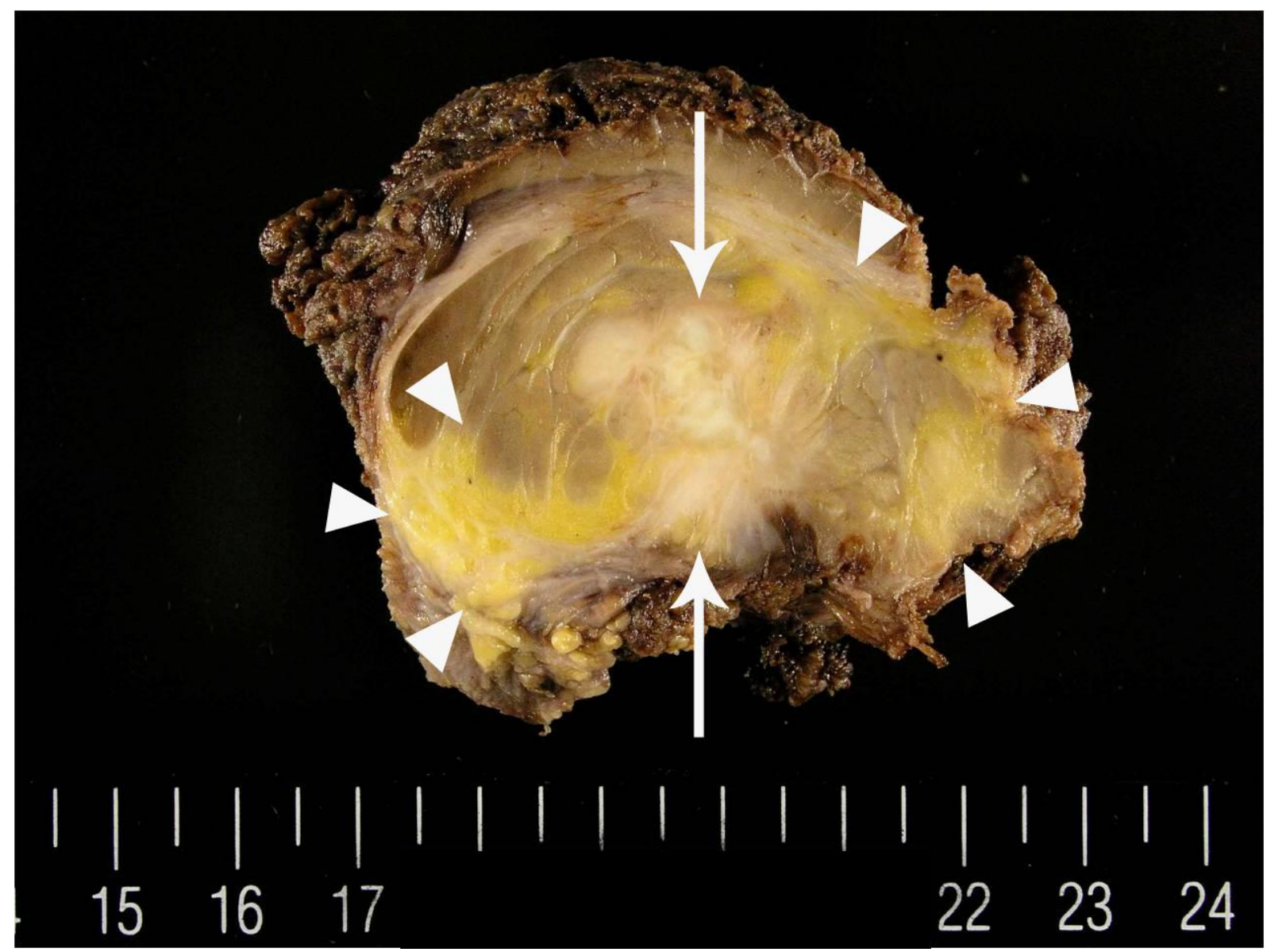


Fig. 2B

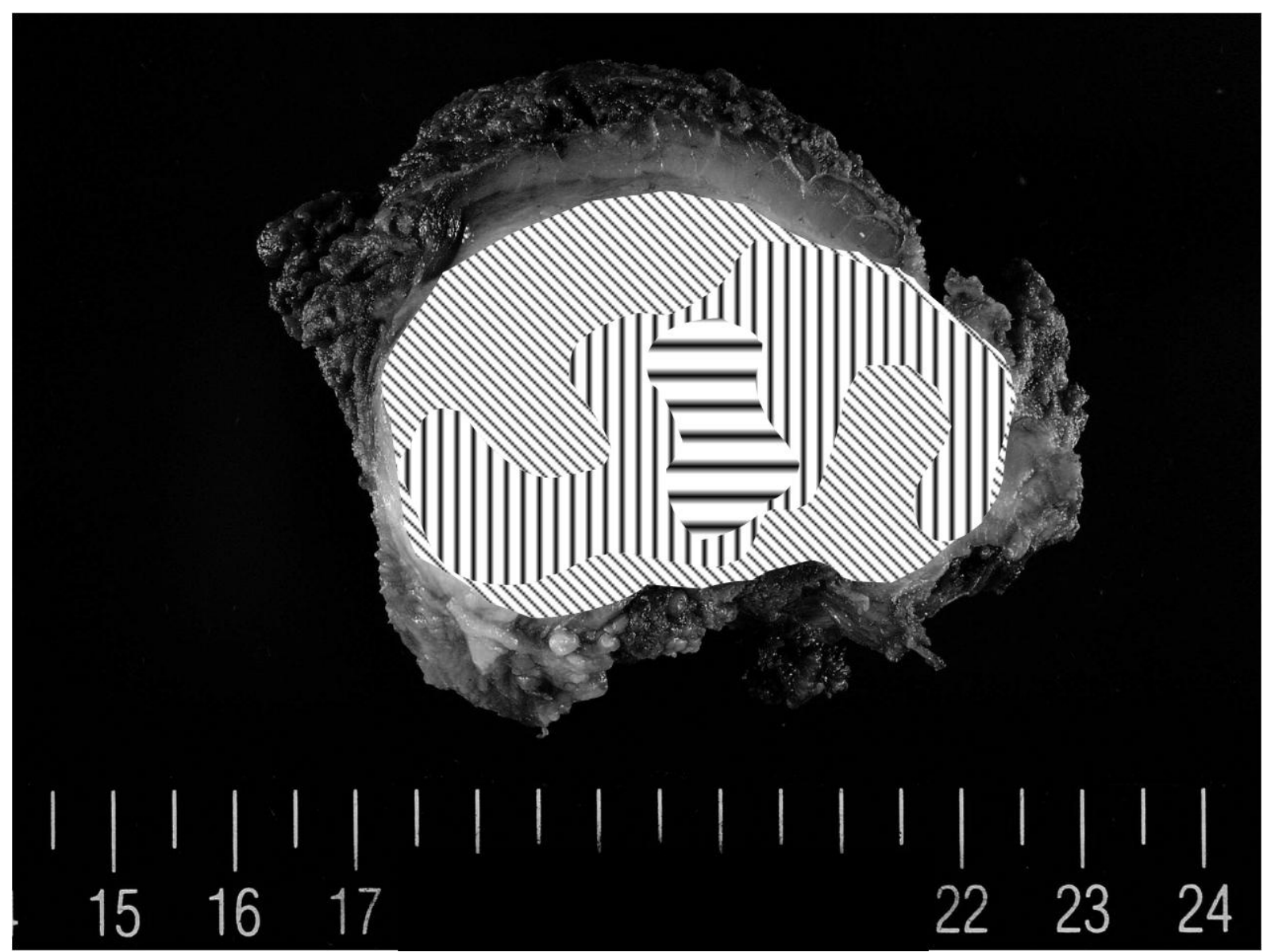


Fig. 3A

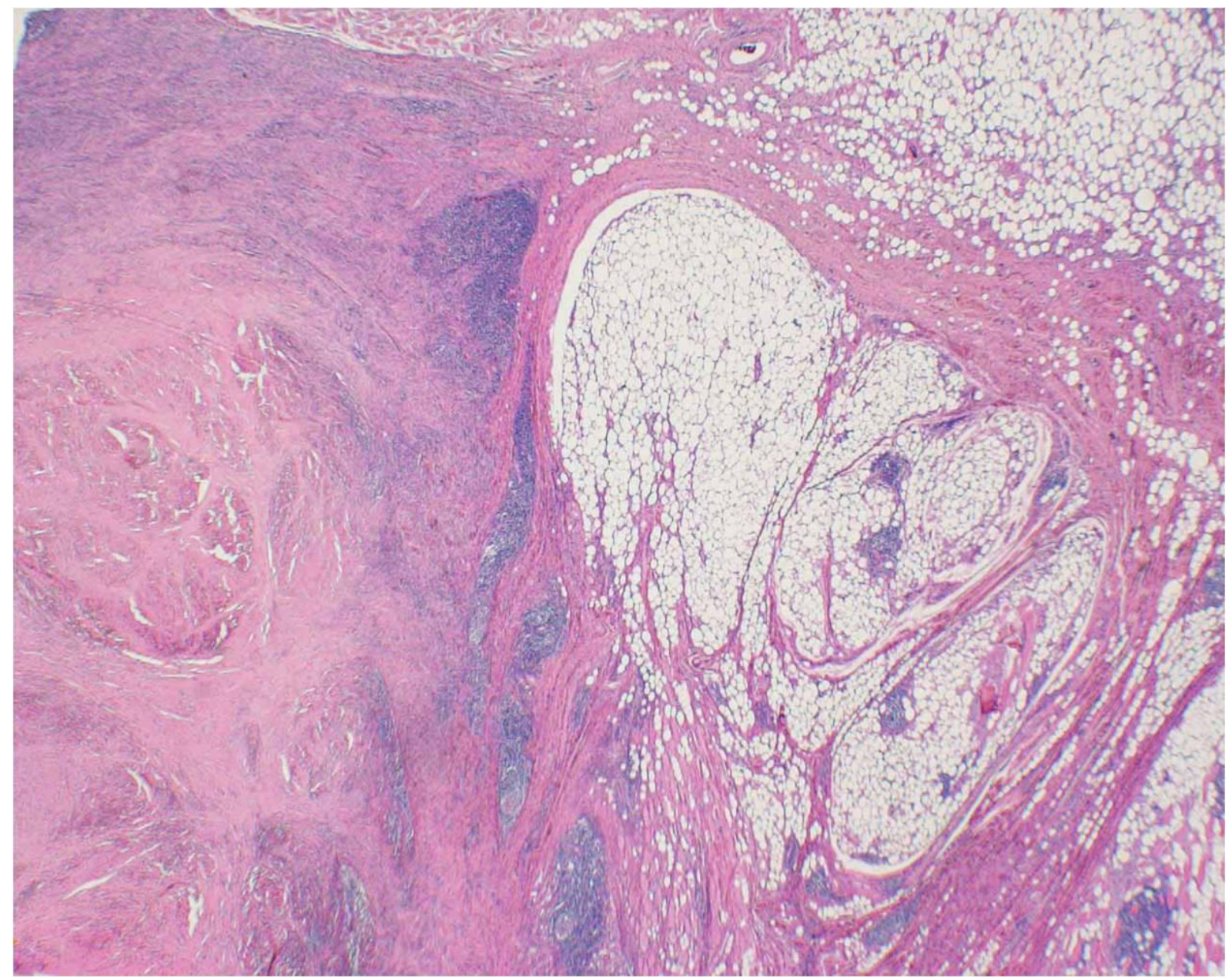


Fig. 3B

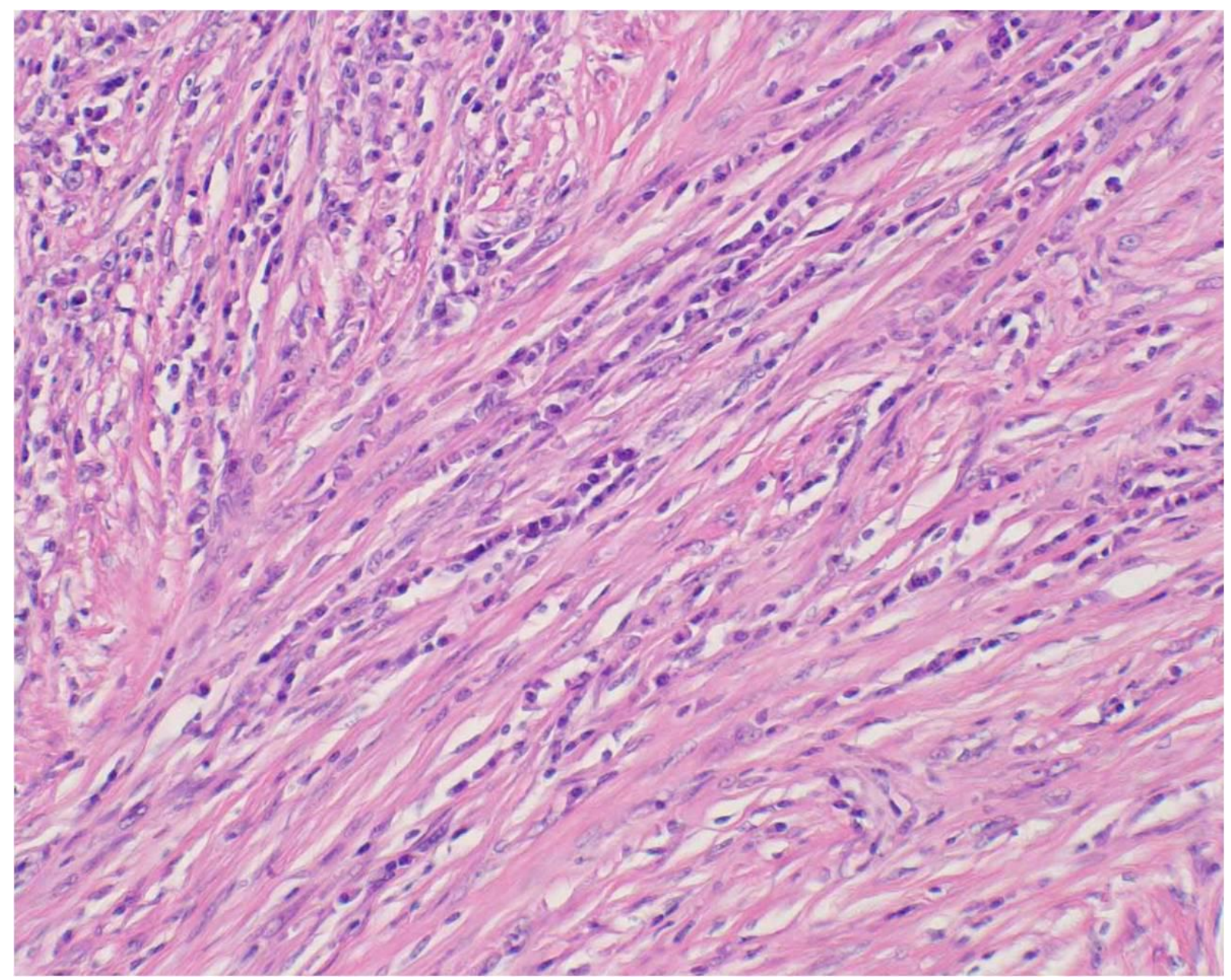


Fig. 3C

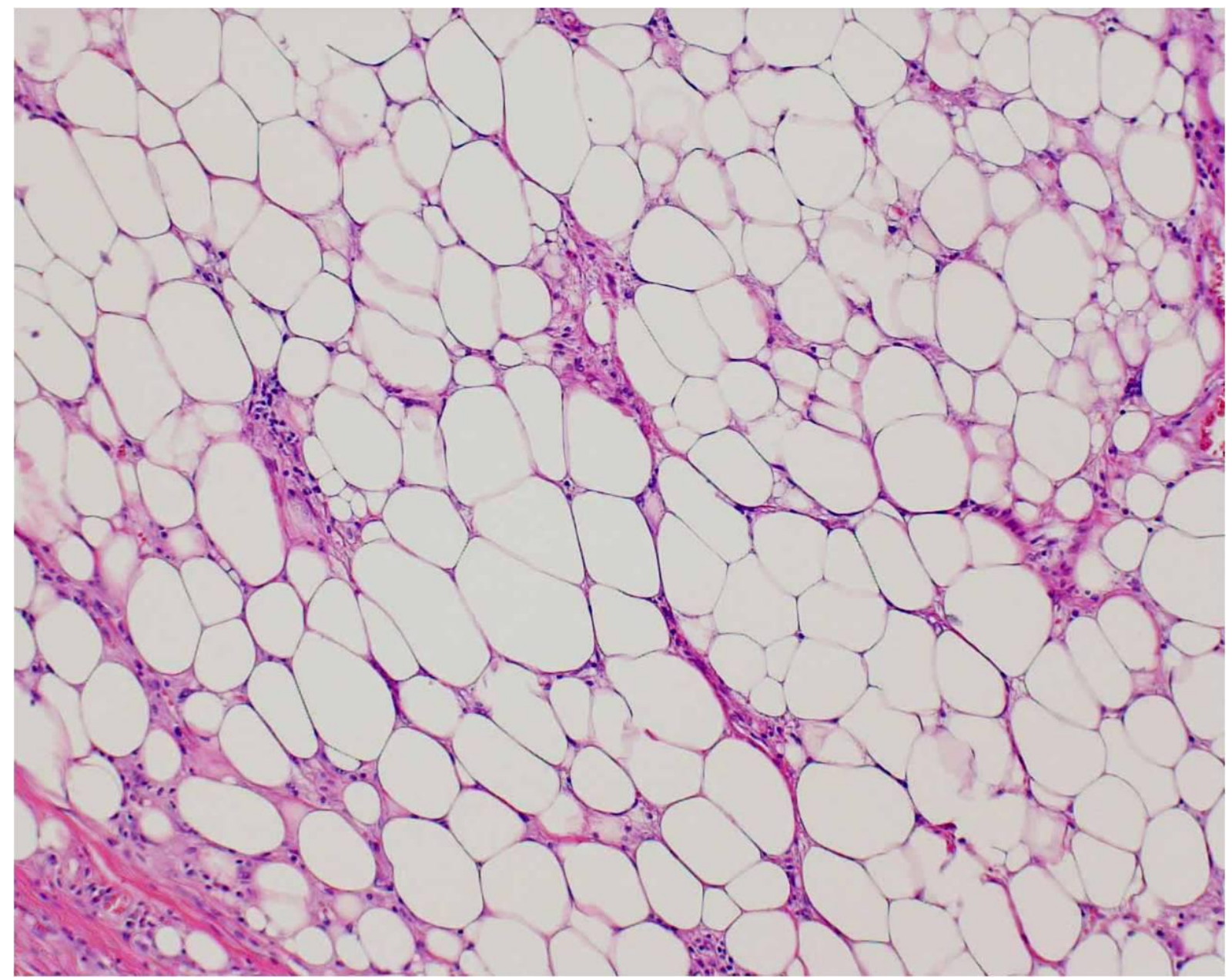


Fig. 3D

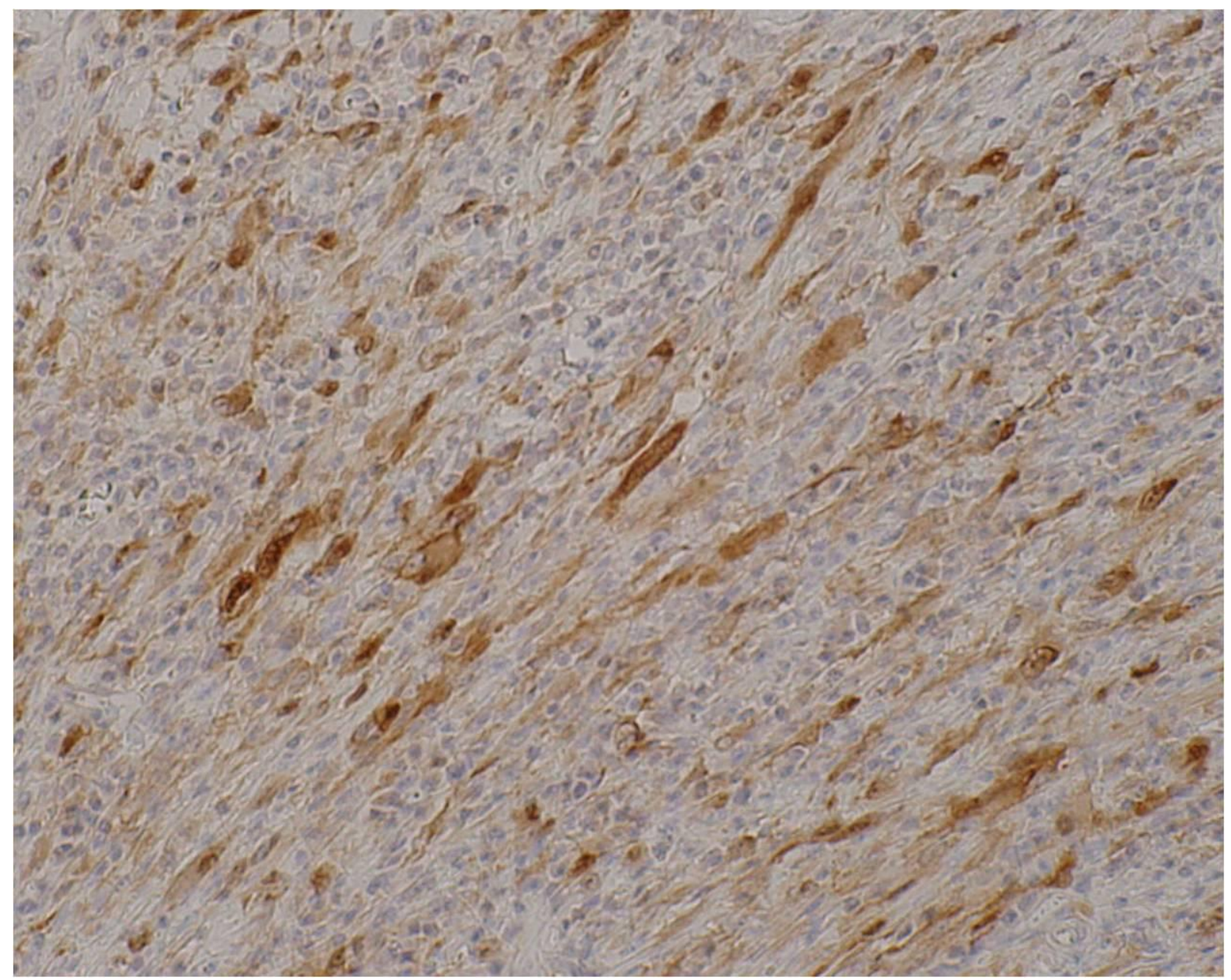

